\title{
The effect of different forms of heparin on point-of-care blood gas analysis
}

\author{
P Sandler, MB ChB, Dip PEC (SA); L N Goldstein, MB BCh, MMed (Emergency Medicine), FCEM (SA), Cert Critical Care (SA) \\ Division of Emergency Medicine, Faculty of Health Sciences, University of the Witwatersrand, Johannesburg, South Africa
}

Corresponding author: L N Goldstein (drg666@gmail.com)

\begin{abstract}
Background. Point-of-care blood gas analysis plays an integral role in the management of critically ill and injured patients presenting to the emergency department (ED). While the use of specially manufactured syringes containing electrolyte-balanced dried heparin is recommended when processing these specimens, alternatives including manually self-prepared syringes washed with liquid heparin or heparin vacutainers are still often used.

Objectives. To assess the effect of two concentrations of liquid heparin and the use of heparin vacutainers on the reliability of blood gas analysis results compared with the recommended standard of dried heparin syringes in the ED setting.

Methods. Blood samples were drawn from 54 patients attending a tertiary-level hospital ED. Individual samples were distributed equally among each of four different collection devices: a dried heparin syringe, self-prepared syringes washed separately with $1000 \mathrm{IU} / \mathrm{mL}$ and $5000 \mathrm{IU} / \mathrm{mL}$ liquid heparin, and a heparin vacutainer. Results obtained from the standard dried heparin syringes were compared with those from the other three methods.

Results. For both the liquid heparin cohorts, partial pressure of carbon dioxide $\left(\mathrm{pCO}_{2}\right)$, potassium $\left(\mathrm{K}^{+}\right)$, sodium $\left(\mathrm{Na}^{+}\right)$, ionised calcium $\left(\mathrm{iCa}{ }^{2+}\right)$ and haemoglobin had $>20 \%$ of results falling beyond the total allowable error. $\mathrm{iCa}^{2+}$ and $\mathrm{K}^{+}$results were most affected in the $5000 \mathrm{IU} / \mathrm{mL}$ cohort and $\mathrm{iCa}^{2+}$ and $\mathrm{Na}^{+}$in the $1000 \mathrm{IU} / \mathrm{ml}$ cohort. $\mathrm{pCO}_{2}, \mathrm{pH}$ and iCa ${ }^{2+}$ were the most significantly affected in the heparin vacutainer cohort. Conclusions. Use of liquid heparin can result in significant negative bias in the majority of blood gas analytes, especially electrolytes. Heparin vacutainer use can result in unacceptable variations in the respiratory analytes. While standard dried heparin syringes may not always be available, it is of vital importance that practitioners be aware of these biases and limitations when using substitutes.
\end{abstract}

S Afr Med J 2018;108(3):224-229. DOI:10.7196/SAMJ.2018.v108i3.12626

Point-of-care blood gas analysis and its interpretation have become an indispensable tool and skill in the management of patients in the emergency department (ED). With numerous indications, arterial or venous blood gas measurement is usually performed on critically ill and injured patients. The results can have a massive influence on the further management of these extremely vulnerable groups of patients. Modern blood gas machines are now available as pointof-care apparatus and are considered to be very accurate, providing information regarding analytes and other parameters extending far beyond the original blood gas variables, with results comparable to those obtained in a laboratory setting. ${ }^{[1]}$

While there are various guidelines regarding the manual preparation of self-prepared heparinised syringes, there is no universal consensus or accepted standard on how best to do this, with many sources differing on various aspects. The concentration of liquid heparin that should be used and the blood sample volume are often not stated. Recommended syringe and needle sizes vary, as does the proposed volume of heparin to be drawn into the syringe. ${ }^{[2-4]}$ Even in the presence of these occasionally nonspecific guidelines, preparation of self-prepared heparinised syringes remains non-standardised among the majority of doctors, even within institutions. ${ }^{[5]}$

The addition of liquid heparin to a blood sample can lead to error through dilution of the sample and binding of heparin to positive ions, resulting in a negative bias. The composition of the liquid heparin itself (e.g. sodium heparin v. heparin calcium) may result in a positive bias. Electrolytes and partial pressure of carbon dioxide $\left(\mathrm{pCO}_{2}\right)$ appear to be the variables most susceptible to dilution, while $\mathrm{pH}$ and partial pressure of oxygen $\left(\mathrm{pO}_{2}\right)$ remain largely unaffected. ${ }^{[5-7]}$ Ionised calcium is the ion most greatly affected by the binding of heparin salts, although sodium and potassium may also be affected. ${ }^{[7,8]}$ These factors, in conjunction with the fact that the preparation of self-prepared heparinised syringes is nonstandardised, means that even repeated blood gas samples from the same patient may not necessarily be comparable or reliable, even if handled by the same operator.

In the light of the above, it is understandable that the Clinical and Laboratory Standards Institute guidelines for blood gas analysis ${ }^{[9]}$ do not describe the blood collecting procedure for self-prepared heparinised syringes, but rather recommend the use of specially manufactured pre-heparinised syringes containing dried heparin, which is preferably electrolyte balanced (dried heparin syringes). They warn against the possible adverse effects of liquid heparin on analysis results. ${ }^{[9]}$ However, the practice of self-preparing syringes continues when there is no access to the recommended dried heparin syringes.

\section{Objective}

To evaluate the effects of different concentrations of liquid heparin and heparin vacutainers on blood gas and electrolyte analysis and to compare these measurements with those obtained using the recommended standard technique (dried heparin syringes).

\section{Methods}

This prospective, cross-sectional study took place in the ED of a tertiary public hospital in Johannesburg, South Africa (SA), from 16 January 2015 to 31 July 2015. Permission to conduct the study was granted by the Human Research Ethics Committee of the University of the Witwatersrand (ref. no. M141182) and the hospital. Patients 
presenting to the ED who were aged $>18$ years, able to give consent and had clinical indications for blood gas analysis (according to the treating doctor) were included in the study.

Blood was drawn according to standard practice guidelines under clean conditions into a standardised plastic non-heparinised syringe. The decision whether to draw an arterial or a venous sample depended on the patient's clinical condition and the treating doctor's discretion. One millilitre of blood was then distributed from this 'master syringe' into each of four separate collection devices: (i) a dried heparin syringe with electrolyte-balanced dried heparin (PICO50; Radiometer, Denmark); (ii) a vacutainer with spray-coated lithium heparin (liquid heparin $68 \mathrm{IU}$ ) (BD, USA); (iii) a $2 \mathrm{~mL}$ plastic syringe (BD) washed with $1000 \mathrm{IU} / \mathrm{mL}$ sodium heparin (Fresenius, Germany) and (iv) a $2 \mathrm{~mL}$ plastic syringe (BD) washed with $5000 \mathrm{IU} /$ $\mathrm{mL}$ sodium heparin (Fresenius).

Self-prepared syringes were primed by drawing $1 \mathrm{~mL}$ of the appropriate concentration of liquid heparin through a $21 \mathrm{G}$ needle, $40 \mathrm{~mm}$ in length (Isigidi Medical Supplies, SA). The plunger was then drawn back completely in order to fully coat the walls of the syringe. The liquid heparin was then expelled with the needle still connected, the plunger being pumped back and forth twice fully and forcefully. The blood sample was then drawn into the recently 'washed' syringe from the 'master syringe' using the $21 \mathrm{G}$ needle already connected to the 'washed' syringe.

The order in which the blood was distributed into the various collection devices and later processed in the blood gas machine was determined by a random-order generator and was different for each patient.

Blood was inserted into the vacutainer using a $21 \mathrm{G}$ needle which was attached to the 'master syringe. The cap was not removed from the vacutainer at any time to avoid any possible contamination with room air. The vacutainer was then inverted eight times to appropriately mix the heparin and blood sample. The blood was then drawn from the vacutainer into a new, clean, appropriately labelled $2 \mathrm{~mL}$ plastic syringe (BD) with a new, clean $21 \mathrm{G}$ needle attached (Isigidi Medical Supplies). The draw volume for the vacutainers used is $4 \mathrm{~mL}$, but only $1 \mathrm{~mL}$ was instilled into the device. This was done with the intention of better reflecting observed practices in the various Johannesburg EDs and to standardise the volume between the various collection devices.

Samples from the four different collection devices were then processed using the resident blood gas machine (ABL800 Flex; Radiometer) as soon as they had all been prepared. All preparation, collection and processing was performed by a single investigator. The resident blood gas machine ran 4-hourly two-point calibrations and routine quality control programmes.

\section{Sample size calculation}

The estimated sample size was calculated separately for each analyte for a two-way analysis of variance with a $p$-value adjusted for multiple comparisons. A significance level of $5 \%$ and power of $80 \%$ was used, while effect sizes were based on the standard deviations (SDs) of the measurements of each analyte (obtained from quality control data). The calculated sample sizes ranged up to 36 . An additional $50 \%$ of samples were included, for a total sample size of 54 , to provide a meaningful Bland-Altman analysis.

\section{Study sample}

A convenience sample of 54 adult patients was enrolled. This took place when the data collector (PS) was present in the ED.

\section{Statistical analysis}

Results for the various analytes from the three experimental devices were recorded and compared with those of the dried heparin syringe (the recommended standard) from the same patient. Table 1 lists the analytes considered, with their names in full and as abbreviations.

Descriptive statistics (mean and SD) were obtained for each of the analytes in each of the experimental groups. The results of the measurements for the heparin $1000 \mathrm{IU} / \mathrm{mL}$, heparin $5000 \mathrm{IU} / \mathrm{mL}$ and heparin vacutainer samples were then compared against the measurements from the dried heparin syringe group (the reference standard) using Bland-Altman methodology. The bias was calculated from the mean of the differences between the experimental group measurement and the standard group measurements. The precision was determined using the $95 \%$ limits of agreement (LOA) of this bias. The experimental samples were compared against the standard group using the paired $t$-test (comparison of means). They were also compared against the total allowable error (TEa), and the percentages of measurements that exceeded the TEa were calculated. The TEa is the total amount of error that can be tolerated without invalidating the medical usefulness of the analytical result. Two methods are considered to give clinically equivalent results if results measured on the same specimen do not differ by more than the specified TEa for that analyte. Specifications for TEa were initially compiled by Ricós et al., ${ }^{[10]}$ with updated figures available online. ${ }^{[11,12]} \mathrm{A}$ TEa was not available for oxygen saturation $\left(\mathrm{sO}_{2}\right)$.

All data were captured onto an electronic spreadsheet (Microsoft Excel 2010; Microsoft, USA). Data were analysed using SAS version 9.3 (SAS Institute, USA). A 5\% significance level was used throughout.

\section{Results}

\section{Patient characteristics}

Samples from 54 patients were used in the study. Venous blood samples accounted for $68.5 \%(n=37)$, while the remainder were arterial blood gas samples.

\section{Sample container device comparison}

The means and SDs obtained for the various analytes via dried heparin syringes are shown in Table 2. Results of the Bland-Altman analyses as well as $95 \%$ LOA and percentages of results falling outside the TEa for the $1000 \mathrm{IU} / \mathrm{mL}, 5000 \mathrm{IU} / \mathrm{mL}$ and heparin vacutainer cohorts are shown in Tables 3, 4 and 5, respectively. Bland-Altman plots for $\mathrm{pCO}_{2}$ and $\mathrm{pO}_{2}$ are presented in Figs 1 and 2, respectively. Bland-Altman plots for the rest of the analytes are presented

\begin{tabular}{ll}
\multicolumn{2}{l}{ Table 1. Analytes considered for comparison } \\
\hline Analyte (units) & Abbreviation \\
\hline $\mathrm{pH}$ & $\mathrm{pH}$ \\
Partial pressure of carbon dioxide $(\mathrm{mmHg})$ & $\mathrm{pCO}_{2}$ \\
Partial pressure of oxygen (mmHg) & $\mathrm{pO}_{2}$ \\
Oxygen saturation (\%) & $\mathrm{SO}_{2}$ \\
Bicarbonate (mmol/L) & $\mathrm{HCO}_{3}$ \\
Base excess (mmol/L) & $\mathrm{BE}^{-}$ \\
Potassium (mmol/L) & $\mathrm{K}^{+}$ \\
Sodium $(\mathrm{mmol} / \mathrm{L})$ & $\mathrm{Na}^{+}$ \\
Ionised calcium $(\mathrm{mmol} / \mathrm{L})$ & $\mathrm{iCa}^{2+}$ \\
Glucose $(\mathrm{mmol} / \mathrm{L})$ & - \\
Lactate $(\mathrm{mmol} / \mathrm{L})$ & - \\
Haemoglobin $(\mathrm{g} / \mathrm{dL})$ & $\mathrm{Hb}$
\end{tabular}


in supplementary Figs 1-12 (these can be obtained from the corresponding author, emaildrg666@gmail.com). The pH, $\mathrm{pO}_{2}$ and $\mathrm{sO}_{2}$ from the two liquid heparin cohorts and potassium $\left(\mathrm{K}^{+}\right)$, glucose,

\begin{tabular}{|c|c|c|c|}
\hline Analyte & $N$ & $n$ excluded & Mean (SD) \\
\hline $\mathrm{pH}$ & 54 & 0 & $7.4(0.1)$ \\
\hline $\mathrm{pCO}_{2}$ & 54 & 0 & $38.3(18.3)$ \\
\hline $\mathrm{pO}_{2}$ & 53 & $1^{*}$ & $51.0(34.3)$ \\
\hline $\mathrm{sO}_{2}$ & 53 & $1^{\dagger}$ & $63.3(31.3)$ \\
\hline $\mathrm{HCO}_{3}^{-}$ & 52 & $2^{\dagger, *}$ & $22.2(4.7)$ \\
\hline $\mathrm{BE}$ & 53 & $1^{*}$ & $-1.8(6.0)$ \\
\hline $\mathrm{K}^{+}$ & 54 & 0 & $4.1(0.7)$ \\
\hline $\mathrm{Na}^{+}$ & 51 & $3^{\dagger}$ & $135.5(6.2)$ \\
\hline Glucose & 54 & 0 & $8.8(7.1)$ \\
\hline $\mathrm{iCa}^{2+}$ & 53 & $1^{\dagger}$ & $1.2(0.2)$ \\
\hline $\mathrm{Hb}$ & 53 & $1^{\dagger}$ & $12.4(3.9)$ \\
\hline Lactate & 53 & $1^{\dagger}$ & $2.4(1.6)$ \\
\hline \multicolumn{4}{|c|}{$\begin{array}{l}\mathrm{pCO}_{2}=\text { partial pressure of carbon dioxide; } \mathrm{pO}_{2}=\text { partial pressure of oxygen; } \mathrm{sO}_{2}=\text { oxygen } \\
\text { saturation; } \mathrm{HCO}=\text { bicarbonate; } \mathrm{BE}=\text { base excess; } \mathrm{K}^{+}=\text {potassium; } \mathrm{Na}^{+}=\text {sodium; } \mathrm{iCa}^{+2}= \\
\text { ionised calcium; } \mathrm{Hb}=\text { haemoglobin; } \mathrm{SD}=\text { standard deviation. } \\
\text { Exclusions due to pre-analytical errors: } \\
\text { Exene dried heparin syringe pO } \mathrm{O}_{2} \text { was not recorded. } \\
\text { 'Excluded due to equipment problems. } \\
\text { "Excluded as spurious. }\end{array}$} \\
\hline
\end{tabular}

haemoglobin $(\mathrm{Hb})$ and lactate results from the heparin vacutainer cohort were statistically significantly different from the results for the standard dried heparin syringe.

\section{Discussion}

Although it has long been known that heparin use may lead to errors in the processing of blood gases, it is still considered the only suitable anticoagulant when performing blood gas analysis. ${ }^{[13,14]}$

The four methods evaluated for collecting blood gases for analysis were representative of the range of methods used in our environment.

\section{Effects of liquid heparin \\ $1000 \mathrm{IU} / \mathrm{mL}$ sodium heparin}

Results for $\mathrm{pO}_{2}, \mathrm{sO}_{2}$ and $\mathrm{pH}$ did not differ from the dried heparin syringe reference sample and could therefore be used reliably in the clinical setting. Although $25.9 \%$ of results for $\mathrm{pCO}_{2}$ were outside the $\mathrm{TEa}$, this would not be considered clinically significant. Respiratory analytes and $\mathrm{pH}$ were therefore not clinically significantly affected by $1000 \mathrm{IU} / \mathrm{mL}$ of sodium heparin.

This concentration of liquid heparin yielded particularly unreliable results for sodium $\left(\mathrm{Na}^{+}\right)$and ionised calcium $\left(\mathrm{iCa}^{2+}\right)$ and to a lesser degree $\mathrm{K}^{+}, \mathrm{Hb}$ and glucose. The same could be said for bicarbonate $\left(\mathrm{HCO}_{3}^{-}\right)$, base excess (BE) and lactate (each of which had $<20 \%$ results beyond the TEa). This is not dissimilar to previous findings. ${ }^{[7,8]}$

Table 3. Sodium heparin $1000 \mathrm{IU} / \mathrm{mL}$

\begin{tabular}{|c|c|c|c|c|c|c|}
\hline Analyte & Mean (SD) & Mean bias (SD) & 95\% LOA & TEa, \% & Cases outside TEa, \% & $p$-value \\
\hline $\mathrm{pH}$ & $7.40(0.10)$ & $0.00(0.01)$ & $-0.03-0.03$ & 3.9 & 16.7 & 0.54 \\
\hline $\mathrm{pCO}_{2}$ & $36.9(17.0)$ & $-1.4(2.5)$ & $-6.2-3.4$ & 5.7 & 25.9 & 0.0002 \\
\hline $\mathrm{pO}_{2}$ & $53.0(35.3)$ & $0.1(3.3)$ & $-6.4-6.5$ & 20.2 & 5.6 & 0.87 \\
\hline $\mathrm{sO}_{2}$ & $64.1(31.9)$ & $-0.1(3.4)$ & $-6.7-6.4$ & * & - & 0.77 \\
\hline $\mathrm{HCO}_{3}^{-}$ & $21.45(4.30)$ & $-0.72(0.53)$ & $-1.76-0.33$ & 5.6 & 9.3 & $<0.0001$ \\
\hline $\mathrm{BE}$ & $-2.76(5.77)$ & $-0.84(0.51)$ & $-1.84-0.15$ & 85 & 18.5 & $<0.0001$ \\
\hline $\mathrm{K}^{+}$ & $3.85(0.56)$ & $-0.16(0.11)$ & $-0.4-0.1$ & 5.6 & 20.4 & $<0.0001$ \\
\hline $\mathrm{Na}^{+}$ & $130.1(6.5)$ & $-5.4(1.7)$ & $-8.76--2.04$ & 0.73 & 90.7 & $<0.0001$ \\
\hline Glucose & $8.50(6.85)$ & $-0.36(0.40)$ & $-1.1-0.4$ & 6.96 & 14.8 & $<0.0001$ \\
\hline $\mathrm{iCa}^{2+}$ & $1.12(0.16)$ & $-0.12(0.05)$ & $-0.21--0.03$ & 2.0 & 94.4 & $<0.0001$ \\
\hline $\mathrm{Hb}$ & $11.9(3.7)$ & $-0.49(0.53)$ & $-1.54-0.55$ & 4.19 & 50 & $<0.0001$ \\
\hline Lactate & $2.24(1.47)$ & $-0.15(0.19)$ & $-0.52-0.23$ & 30.4 & 3.7 & $<0.0001$ \\
\hline
\end{tabular}

Table 4. Sodium heparin $5000 \mathrm{IU} / \mathrm{mL}$

\begin{tabular}{|c|c|c|c|c|c|c|}
\hline Analyte & Mean (SD) & Mean bias (SD) & 95\% LOA & TEa, \% & Cases outside TEa, \% & $p$-value \\
\hline $\mathrm{pH}$ & $7.40(0.09)$ & $0.00(0.01)$ & $-0.03-0.02$ & 3.9 & 13.0 & 0.39 \\
\hline $\mathrm{pCO}_{2}$ & $36.9(16.9)$ & $-1.3(2.6)$ & $-6.4-3.8$ & 5.7 & 27.8 & 0.0004 \\
\hline $\mathrm{pO}_{2}$ & $51.7(36.4)$ & $0.8(3.9)$ & $-7.0-8.5$ & 20.2 & 5.6 & 0.17 \\
\hline $\mathrm{sO}_{2}$ & $63.1(32.2)$ & $-0.2(3.0)$ & $-6.1-5.7$ & * & - & 0.60 \\
\hline $\mathrm{HCO}_{3}^{-}$ & $21.45(4.46)$ & $-0.74(0.46)$ & $-1.64-0.16$ & 5.6 & 9.3 & $<0.0001$ \\
\hline $\mathrm{BE}$ & $-2.59(5.69)$ & $-0.72(2.31)$ & $-2.86-0.86$ & 85 & 25.9 & 0.027 \\
\hline $\mathrm{K}^{+}$ & $3.78(0.64)$ & $-0.28(0.15)$ & $-0.6-0.0$ & 5.6 & 55.6 & $<0.0001$ \\
\hline $\mathrm{Na}^{+}$ & $134.2(6.0)$ & $-1.3(1.0)$ & $-3.21-0.70$ & 0.73 & 61.1 & $<0.0001$ \\
\hline Glucose & $8.44(6.62)$ & $-0.36(0.58)$ & $-1.5-0.8$ & 6.96 & 14.8 & $<0.0001$ \\
\hline $\mathrm{iCa}^{2+}$ & $0.92(0.17)$ & $-0.32(0.11)$ & $-0.54--0.10$ & 2.0 & 98.2 & $<0.0001$ \\
\hline $\mathrm{Hb}$ & $11.9(3.7)$ & $-0.47(0.32)$ & $-1.09-0.16$ & 4.19 & 38.9 & $<0.0001$ \\
\hline Lactate & $2.25(1.48)$ & $-0.14(0.25)$ & $-0.64-0.36$ & 30.4 & 3.7 & $<0.0001$ \\
\hline
\end{tabular}




\begin{tabular}{|c|c|c|c|c|c|c|}
\hline Analyte & Mean (SD) & Mean bias (SD) & 95\% LOA & TEa, \% & Cases outside TEa, \% & $p$-value \\
\hline $\mathrm{pH}$ & $7.45(0.10)$ & $0.05(0.03)$ & $0.00-0.10$ & 3.9 & 92.6 & $<0.0001$ \\
\hline $\mathrm{pCO}_{2}$ & $31.8(14.6)$ & $-6.5(4.8)$ & $-15.8-2.8$ & 5.7 & 100 & $<0.0001$ \\
\hline $\mathrm{pO}_{2}$ & $55.7(31.2)$ & $4.4(10.9)$ & $-16.9-25.6$ & 20.2 & 18.5 & 0.0052 \\
\hline $\mathrm{sO}_{2}$ & $71.2(27.0)$ & $7.8(6.8)$ & $-5.5-21.1$ & * & - & $<0.0001$ \\
\hline $\mathrm{HCO}_{3}^{-}$ & $21.90(4.75)$ & $-0.29(0.89)$ & $-2.03-1.45$ & 5.6 & 18.5 & $<0.0001$ \\
\hline $\mathrm{BE}$ & $-2.47(5.97)$ & $-0.60(0.56)$ & $-1.7-0.5$ & 85 & 20.4 & $<0.0001$ \\
\hline $\mathrm{K}^{+}$ & $4.05(0.71)$ & $-0.01(0.08)$ & $-0.2-0.1$ & 5.6 & 1.9 & 0.48 \\
\hline $\mathrm{Na}^{+}$ & $134.4(6.4)$ & $-1.1(0.8)$ & $-2.59-0.43$ & 0.73 & 53.7 & $<0.0001$ \\
\hline Glucose & $8.72(6.90)$ & $-0.07(0.36)$ & $-0.8-0.6$ & 6.96 & 1.9 & 0.14 \\
\hline $\mathrm{iCa}^{2+}$ & $1.14(0.15)$ & $-0.10(0.03)$ & $-0.16--0.04$ & 2.0 & 98.2 & 0.032 \\
\hline $\mathrm{Hb}$ & $12.4(3.9)$ & $0.07(0.32)$ & $-0.55-0.69$ & 4.19 & 7.4 & 0.12 \\
\hline Lactate & $2.37(1.54)$ & $-0.02(0.21)$ & $-0.44-0.39$ & 30.4 & 0.0 & 0.48 \\
\hline
\end{tabular}

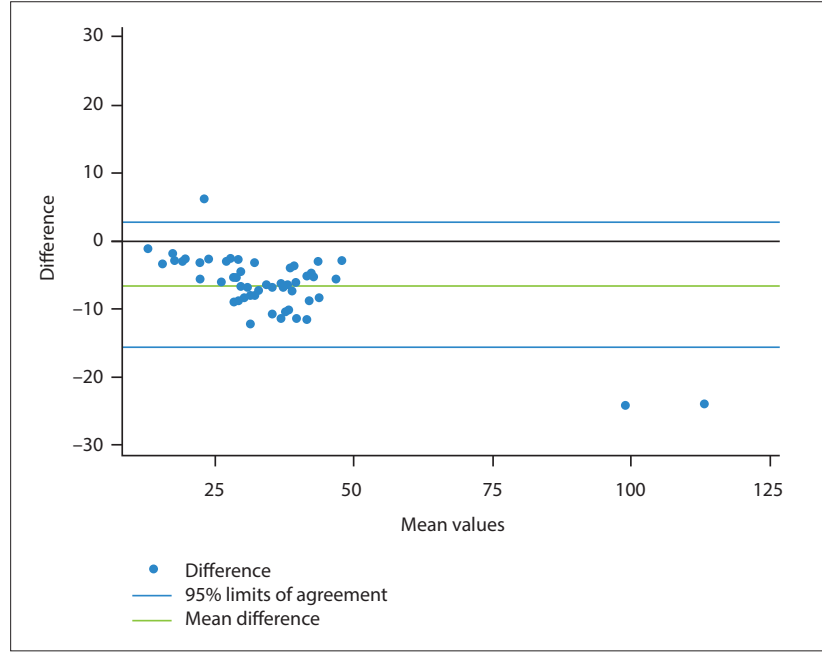

Fig. 1. Bland-Altman plot for partial pressure of carbon dioxide $(\mathrm{mmHg})$ in a heparin vacutainer compared with the standard dried heparin syringe.

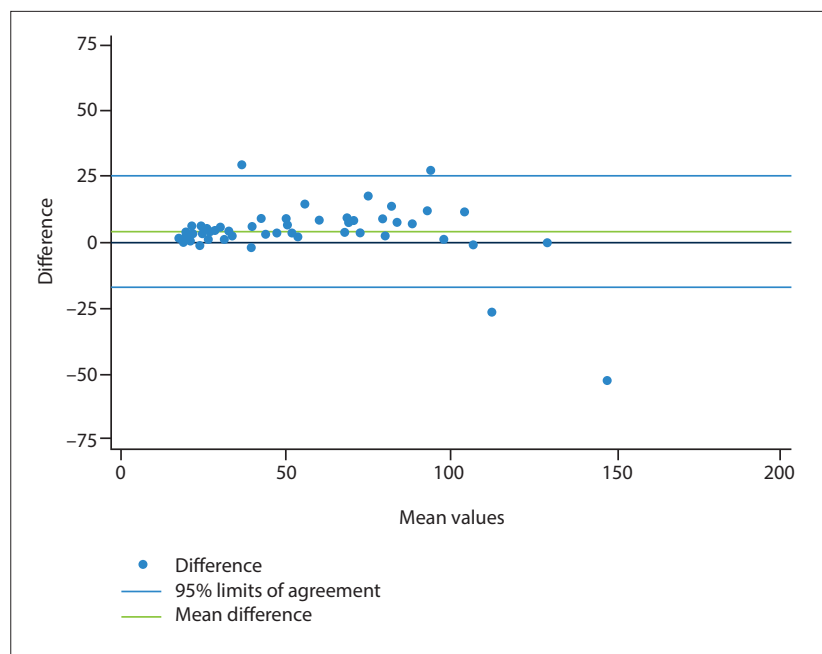

Fig. 2. Bland-Altman plot for partial pressure of oxygen ( $\mathrm{mmHg}$ ) in a heparin vacutainer compared with the standard dried heparin syringe.

\section{$5000 \mathrm{IU} / \mathrm{mL}$ sodium heparin}

Similar to the $1000 \mathrm{IU} / \mathrm{mL}$ dilution of liquid heparin, the electrolytes in the $5000 \mathrm{IU} / \mathrm{mL}$ samples had their main statistically significant differences in the electrolytes. $\mathrm{iCa}^{2+}$ and $\mathrm{K}^{+}$were affected more than in the $1000 \mathrm{IU} / \mathrm{mL}$ cohort, presumably owing to the greater concentration of heparin resulting in a greater amount of heparin salts able to bind those positive ions. Conversely, $\mathrm{Na}^{+}$was affected less than in the $1000 \mathrm{IU} / \mathrm{mL}$ cohort, probably owing to the greater concentration of $\mathrm{Na}^{+}$being added to the sample (from the liquid sodium heparin) in part compensating for the negative bias and outweighing the increased amount of binding with the $\mathrm{Na}^{+}$that would be taking place. The remainder of the analytes exhibited similar findings to those of the $1000 \mathrm{IU} / \mathrm{mL}$ cohort, suggesting that any bias present is independent of heparin concentration and more likely due to dilution, as has been noted previously. ${ }^{[5-7]}$

\section{Effects of the heparin vacutainer}

The respiratory analytes as well as the $\mathrm{pH}$ were significantly affected when evaluated from the heparin vacutainer. Every effort was made not to break the seal of the vacutainer or to introduce exogenous sources of contamination (i.e. air bubbles), but the values measured compared with the dried heparin syringe sample suggest that these respiratory values were biased towards atmospheric conditions (in Johannesburg $\mathrm{pO}_{2} \sim 134 \mathrm{mmHg}, \mathrm{pCO}_{2} \sim 0.3 \mathrm{mmHg}$ ). The $\mathrm{pCO}_{2}$ was almost uniformly decreased (with an apparent greater degree of variation seen in higher values) and the $\mathrm{pO}_{2}$ trended towards values of $100-130 \mathrm{mmHg}$ with a wide variability suggested by the limits of agreement (Figs 1 and 2). This study was underpowered to adequately determine the validity of this hypothesis. The effect on $\mathrm{pH}$ was more marked, probably as a consequence of the changes in $\mathrm{pCO}_{2}$ values. This alteration in $\mathrm{pH}$ may also account for some of the electrolyte biases noted. ${ }^{[15,16]} \mathrm{Na}^{+}$was lowered in a similar magnitude to that in the $5000 \mathrm{IU} / \mathrm{ml}$ cohort, while $\mathrm{iCa}^{2+}$ was similar to that in the $1000 \mathrm{IU} / \mathrm{ml}$ cohort. The results for $\mathrm{BE}$ and $\mathrm{HCO}_{3}{ }^{-}$were similar to those of the dried heparin syringe cohorts. Findings for $\mathrm{K}^{+}$, glucose, $\mathrm{Hb}$ and lactate revealed no statistically relevant difference in results. There was also a trend towards narrower limits of agreement compared with the liquid heparins (apart from the respiratory variables), presumably owing to the fixed amount of heparin v. a more changeable volume in the liquid heparin cohorts.

Vacutainers containing spray-coated lithium heparin contain a controlled, known amount of heparin (68 IU), and dilution was therefore not a problem. However, there is significant 'dead space' in the vacutainer if it is not filled adequately, which if contaminated with air could significantly skew the actual gas parameters, i.e. $\mathrm{pO}_{2}$, $\mathrm{pCO}_{2}$, etc. There is a paucity of data available regarding the use of 
vacutainers for the processing of blood gases, with only one study ${ }^{[17]}$ comparing the measurement of $\mathrm{iCa}^{2+}$ in lithium heparin-containing blood tubes v. dried heparin syringes. Those investigators found a slightly lower mean bias of results in the vacutainers.

\section{Reliability in the clinical setting}

Both concentrations of liquid heparin had a significant effect on electrolyte chemistry as well as $\mathrm{Hb}$ and lactate, but did not greatly affect the respiratory analytes or $\mathrm{pH}$. Conversely, the heparin vacutainer results indicated more electrolyte stability but various effects on the respiratory analytes and the $\mathrm{pH}$.

The reliability of blood gas results obtained via the different methods has different implications in the laboratory setting v. the clinical setting. The extent to which values obtained fall outside the TEa is important in terms of reliability of these results in a laboratory setting. However, a more telling question for clinicians is whether these biased results in any way adversely affect decisions on patient management.

These 'abnormalities' could potentially have the most detrimental consequences in a scenario involving inexperienced or junior doctors. A junior doctor's zealousness to treat an 'abnormal' result could mean that he or she treats the numbers rather than considering the patient and whether or not the results are in keeping with the patient's presentation (more experienced doctors may not be immune to this tendency). These errors would most be likely to occur with analytes the real values of which are situated at the lower levels of normal or slightly higher than normal, as overall there appears to be a negative biasing of results - a lower level of normal will become abnormally low, and mildly high yet abnormal may become 'normalised'. Examples may include initiating intravenous replacement of potassium at levels $<3 \mathrm{mmol} / \mathrm{L}$, transfusing blood to patients with an $\mathrm{Hb}<7 \mathrm{~g} / \mathrm{dL}$ (practices that are not without significant risks) or missing a raised $\mathrm{iCa}^{2+}$ because it has been erroneously lowered into the normal range, and therefore possibly not considering hypercalcaemia in the patient's differential diagnosis.

It is also known that the greater the volume of heparin present, the larger the bias will be. The fact remains that self-prepared syringe techniques are poorly standardised, and the investigators have observed that at times far greater biases may be obtained because of this, leading to very significantly skewed results with potentially deleterious clinical consequences.

\section{Study limitations}

The resident Radiometer blood gas machine was replaced on two occasions with different models (ABL700, ABL800 Basic) as a result of prearranged repair plans. None of these machines measured chloride, an analyte that is important when considering acid-base status (i.e. anion gap, strong ion difference.)

Dilution of the sample is not a feature unique to liquid heparin, as the addition of any liquid with a different composition to whole blood (e.g. normal saline) would have a diluting effect. It has been found (as would be expected) that the greater the added volume of liquid heparin, the greater the discrepancy in results. ${ }^{[5,18]}$ In order to try to control for the effect of dilution, $1 \mathrm{~mL}$ of blood was used for all the methods. This $1 \mathrm{~mL}$ measurement was not performed with a pipette but with a regular syringe, which meant that there could have been small differences in the amounts. As this was a pragmatic study, the potential difference in amount of blood between patients was not considered to be significant.

Every effort was made not to break the seal of the vacutainer or to introduce exogenous sources of contamination such as air bubbles, but the values measured compared with the dried heparin syringe sample suggest that these respiratory values were biased towards atmospheric conditions.

\section{Conclusions}

The results of this study have highlighted the effects of different concentrations of liquid heparin and heparin vacutainers on blood gas and electrolyte analysis compared with measurements obtained when using the recommended standard technique of a dried heparin syringe. Liquid heparins mainly affected the electrolyte results, especially $\mathrm{iCa}^{2+}$. This inaccuracy was exaggerated at higher concentrations of liquid heparin. Heparin vacutainers tended to maintain electrolyte integrity, but had significant effects on the respiratory analytes and $\mathrm{pH}$.

The electrolyte integrity of heparin vacutainers could have been due to the fixed amount of heparin. Increasing the volume of blood added to the tube could potentially have resulted in even smaller biases in the presence of a more predictable effect, but further studies would need to be done in this area.

The majority of analytes exhibited a negative bias. In the absence of other possible confounders, results obtained when using a heparin vacutainer or liquid heparin that appear elevated are therefore likely actually to be elevated, and perhaps elevated to an even greater degree than they seem to be.

We recommend that wherever possible the recommended dried heparin syringe should be used. However, if these are not available (and until stricter, more specific guidelines are put in place), a low concentration of liquid heparin (preferably $1000 \mathrm{IU} / \mathrm{mL}$ ) in a selfprepared syringe can be used. An alternative, if electrolytes or $\mathrm{Hb}$ are of particular interest, is to use a heparin vacutainer filled with blood to the recommended draw volume, knowing that the respiratory analytes will not be reliable.

Irrespective of the concentration or form of heparin, it is of utmost importance to be aware of the possible biases and relative unreliability of results, especially when translating these results into management of a patient.

Acknowledgements. Radiometer sponsored printing paper for the blood gas machines. We are grateful to Prof. Mike Wells for his general and statistical support.

Author contributions. Both authors contributed substantially to the conceptualisation, design, analysis and interpretation of data. PS drafted the initial version of the manuscript. Both authors revised and edited the manuscript. Final approval of the version to be published was given by both authors.

Funding. None.

Conflicts of interest. None.

1. Mirzazadeh M, Morovat A, James T, Smith I, Kirby I, Shine B. Point-of-care testing of electrolytes and

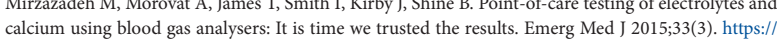
calcium using blood gas analysers: It is
doi.org/10.1136/emermed-2015-204669

2. Burnett RW, Covington AK, Fogh-Andersen N, et al. Approved IFCC recommendations on whole blood sampling, transport and storage for simultaneous determination of $\mathrm{pH}$, blood gases and electrolytes. Eur J Clin Chem Clin Biochem 1995;33(4):247-253.

3. Roberts JR. Arterial puncture and cannulation. In: Roberts and Hedges' Clinical Procedures in Emergency Medicine. 6th ed. Philadelphia, Penn.: Elsevier Saunders, 2014: 369-370.

4. Radiometer Medical ApS. ABL800 Flex Operator's Manual. Copenhagen: Radiometer, 2012 [updated 2012]. http://www.radiometeramerica.com (accessed 21 November 2015).

5. Küme T, Sişman AR, Solak A, Tuğlu B, Cinkooğlu B, Coker C. The effects of different syringe volume, needle size and sample volume on blood gas analysis in syringes washed with heparin. Biochem Med (Zagreb) 2012;22(2):189-201. https://doi.org/10.11613/bm.2012.022

6. Higgins C. The use of heparin in preparing samples for blood gas analysis. MLO Med Lab Obs 2007;39(10):16-18.

Malley WJ. Blood gas sampling errors. In: Clinical Blood Gases: Assessment and Intervention. 2nd ed. St Louis, Miss.: Elsevier Saunders, 2005: 75.

8. Chhapola V, Kumar S, Goyal P. Is liquid heparin comparable to dry balanced heparin for blood gas sampling in intensive care unit? Indian J Crit Care Med 2014;18(1):14-20. https://doi. gas sampling in intensive care
org/10.4103/0972-5229.125428

9. Clinical and Laboratory Standards Institute. Document C46-A: Blood Gas and $\mathrm{pH}$ Analysis and Related Measurements. Wayne, Penn.: NCCLS/CLSI, 2001. 
10. Ricós C, Alvarez V, Cava F, et al. Current databases on biological variation: Pros, cons and progress. Scand J Clin Lab Invest 1999;59(7):491-500. https://doi.org/10.1080/00365519950185229

1. Westgard QC. Desirable Biological Variation Database Specifications. Madison, Wis.: Westgard QC 2009 [updated 2014]. http://www.westgard.com/biodatabasel.htm (accessed 23 May 2017).

12. Data Innovations. Allowable Total Error Table. South Burlington, Vt: Data Innovations LLC, 2015

[updated 2015]. http://datainnovations.com/allowable-total-error-table (accessed 23 May 2017).

3. Hamilton R, Crockett A, Alpers J. Arterial blood gas analysis: Potential errors due to the addition of heparin. Anaesth Intensive Care 1978;6(3):251-256.

4. Clinical and Laboratory Standards Institute. Blood Gas and $\mathrm{pH}$ Analysis and Related Measurements: Approved Guideline. NCCLS Document C46-A2. Wayne, Penn.: CLSI, 2009.

15. Adrogué $\mathrm{HJ}$. Madias NE. Changes in plasma potassium concentration during acute acid-base disturbances. Am J Med 1981;71(3):456-457. https://doi.org/10.1016/0002-9343(81)90182-0
16. Wang S, McDonnell EH, Sedor FA, Toffaletti JG. pH effects on measurements of ionized calcium and nized magnesium in blood. Arch Pathol Lab Med 2002;126(8):947-950.

17. Haverstick DM, Brill LB 2nd, Scott MG, et al. Preanalytical variables in measurement of free (ionized) calcium in lithium heparin-containing blood collection tubes. Clin Chim Acta 2009;403(1-2):102-104. ttps://doi.org/10.1016/i.cca.2009.01.026

18. Chhapola V, Kumar S, Goyal P, et al. Use of liquid heparin for blood gas sampling in pediatric intensive care unit: A comparative study of effects of varying volumes of heparin on blood gas parameters. Indian J Crit Care Med 2013;17(6):350-354. https://doi.org/10.4103/0972-5229.123443

Accepted 2 October 2017. 